\title{
A protocol for evaluating the accuracy of 3D body scanners
}

\author{
Makiko Kouchi ${ }^{\mathrm{a},{ }^{*}}$, Masaaki Mochimaru ${ }^{\mathrm{a}}$, Bruce Bradtmiller ${ }^{\mathrm{b}}$, Hein Daanen ${ }^{\mathrm{c}}, \mathrm{Peng} \mathrm{Li}^{\mathrm{d}}$, Beatriz Nacher ${ }^{\mathrm{e}}$, \\ and Yunja Nam ${ }^{\mathrm{f}}$ \\ ${ }^{\mathrm{a}}$ Digital Human Research Center, National Institute of Advanced Industrial Science and Technology, 2-3-26 \\ Aomi, Koto-ku, Tokyo 135-0064, Japan \\ ${ }^{\mathrm{b}}$ Anthrotech Inc. 503 Xenia Avenue, Yellow Springs, OH 45387, U.S.A. \\ ${ }^{\mathrm{c}}$ Department of Human Performance, TNO Defence, Security and Safety, Business Unit of Human Factors, PO \\ Box 23, 3769ZG Soesterberg, The Netherlands \\ ${ }^{\mathrm{d}}$ Ergonomics Team, WarSTAR, US Army Natick Soldier Research Development \& Engineering Center, Natick MA \\ 01760, U.S.A. \\ ${ }^{\mathrm{e}}$ Instituto de Biomecánica de Valencia, Universidad Politecnica de Valencia, Edificio 9C, Camino de Vera s/n E- \\ 46022 Valencia, Spain \\ ${ }^{\mathrm{f}}$ Department of Clothing and Textiles, College of Human Ecology, Seoul National University, San 56-1, Shillim- \\ Dong, Kwanak-gu, Seoul 151-742, Korea
}

\begin{abstract}
Scan-derived landmarks locations and surface shapes are more and more used, but there is no commonly accepted protocol for evaluating the accuracy of these measurements. Therefore we propose a protocol for evaluating the accuracy of surface shape and the repeatability of scan-derived landmark locations. According to existing Japanese and German domestic standards, we propose to use an artefact (e.g. sphere with diameter of about $120 \mathrm{~mm}$ ) calibrated very accurately for evaluating the accuracy of scanner-systems. For evaluating the repeatability of landmark locations, we propose to use an anthropomorphic dummy with landmark locations premarked. These test objects are measured by a 3D body scanner to be evaluated. Evaluation parameters such as trueness, precision, and repeatability are calculated from the measured data. A round-robin test was conducted in six different institutes using $17 \mathrm{body} / \mathrm{head} /$ foot scanners produced by eight companies. The purposes of the roundrobin test were to evaluate the availability of test objects to different body scanners, and to examine the measurement locations of test objects and quality parameters to be reported. As a result, the proposed test objects could be measured and the data exported by all scanner systems except one, which could not export the ball measurement. For a comparative purpose, a figure of measured surface might be useful.
\end{abstract}

Keywords: Reliability, accuracy, 3D anthropometry, landmarks

\section{Introduction}

Quality of scan-derived anthropometric measurements is one of a main concern for users of body scanners. Recently, 3D body scanners have been more and more used in anthropometric surveys [1,2], but it has been reported that scan-derived body dimensions are not always comparable with those obtained by traditional methods $[3,4]$. Combining scanderived $1 \mathrm{D}$ measurements and those obtained by the traditional methods to make one database may cause problems in terms of the comparability of the data. To avoid this problem, an international standard ISO 20685[5] has been developed to establish a protocol for evaluating the comparability between scanderived 1D measurements and those obtained by the traditional methods. However, landmark locations and body shape can also be obtained by a body scanner, and recently these types of data have become more and more utilized through homologous body

*Corresponding author. E-mail: m-kouchi@aist.go.jp. 
shape modeling [6,7]. ISO TC159/SC3/WG1 has been working to develop international standards related to the quality control of anthropometric data. Considering the present situation, ISO TC159/SC3/WG1 plans to make an international standard that establishes a protocol for evaluating the accuracy of 3D shape measurements and repeatability of landmark locations. The accuracy of scanderived measurements is affected by more factors than that of traditional measurements as shown in Fig. 1. In a tentative protocol, two types of test objects are used: a ball is used to evaluate the accuracy of a scanner system as a tool including both hardware and software; and a dummy is used to evaluate the repeatability of landmark locations. To validate this tentative protocol, we conducted a round-robin test using these test objects. The purposes the round-robin test were to evaluate the availability of test objects to different body scanners, and to obtain materials for discussion on the locations of measurement of test object, quality parameters to represent the scanner performance to be reported. In this paper, we present the results of the round-robin test, and evaluate the tentative protocol for evaluating the accuracy of $3 \mathrm{D}$ body scanners.

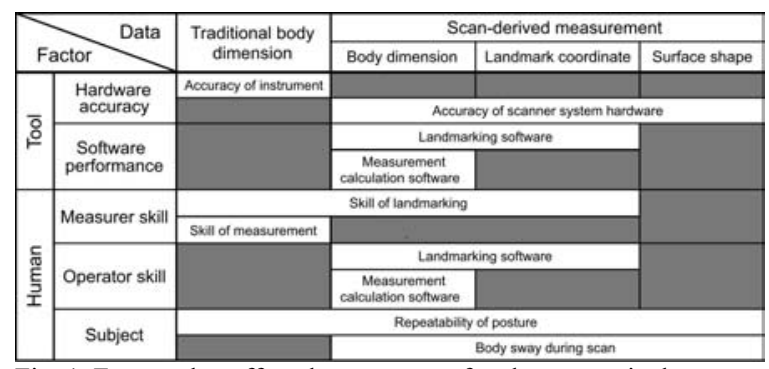

Fig. 1. Factors that affect the accuracy of anthropometric data.

\section{Artefacts}

\subsection{Ball}

There are Japanese and German domestic standards that have established methods of acceptance test and reverification of optical 3D measuring systems. In these standards, ball, ball-bars, and rectangular parallelepipeds are used [8-11]. International standardization of this protocol is in progress by ISO/TC122. Since body scanners are included in optical 3D measuring systems, it is reasonable to use test objects used in these standards. In the present protocol, a ball with diameter of $120.0159 \mathrm{~mm}$ (sphericity $=22.1 \mu \mathrm{m}$ ) was used as one of the test objects. The artefact is a hollow steel ball, and the surface was blasted and treated with TiN to diffuse the reflection (Fig. 2). This treatment was the most successful in a round-robin test conducted in Japan in which balls of several different surface treatments were measured by different 3D measuring systems [12]. The diameter of the ball was determined based on the human body size, cost of manufacturing, and ease of handling.

The ball was calibrated in National Metrology Institute of Japan, AIST, using a coordinate measuring machine (CMM). Only this way, measurement by a $3 \mathrm{D}$ scanner can be traceable to the international standard of the length.

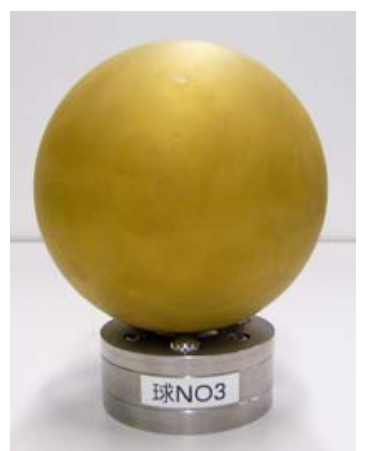

Fig. 2. Artefact: ball $(\varnothing=120.0159 \mathrm{~mm}$, sphericity $=22.1 \mu \mathrm{m}$

\subsection{Dummy}

We used a whole body dummy and a head dummy as described below. We did not use a foot dummy.

\subsubsection{Whole body dummy}

One of the characteristics of body scanners is that they usually accompany with software to detect marker locations or calculate landmark locations. To evaluate landmark locations, an artefact similar to the actual human body is necessary. We used a whole body dummy with the posture recommended in ISO 20685. It is made of FRP (fiber reinforced plastic), with no movable parts such as joints, and has average body dimensions of Japanese females in their 20s (Fig. 3, left) (Nanasai Co. Ltd.) Locations of landmarks are marked with small dents.

\subsubsection{Head dummy}

For the head dummy, we used a dummy shown in Fig. 3, right. The form of the dummy does not reflect the average head form, but has been created so that it has difficult shape features to measure (Nanasai Co. Ltd.) Locations of landmarks are marked with small black dots. 


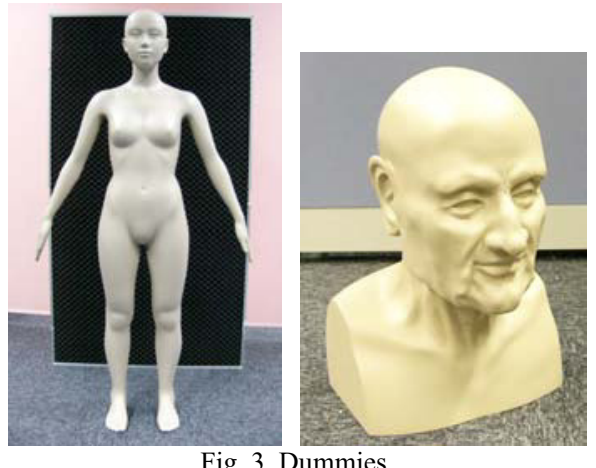

Fig. 3. Dummies.

\section{Measurement of the ball}

\subsection{Measurement locations of the ball}

\subsubsection{Whole body scanner}

In existing standards $[8,10]$, a test object is measured at 5 or 7 different locations. Example is the center and four locations at margin of scanning volume. However, in body scanners, only part of the scanning volume is used because only humans are measured. In the present protocol, we should obtain the data for deciding which locations are appropriate for evaluating a whole body scanner. Therefore, the nine locations shown in Fig. 4A are used. In determining these locations, we assumed that the subject stands with arms and legs abducted as recommended in ISO 20685. Accuracy of the ball placement is within \pm 30 $\mathrm{mm}$ of the target position for height, lateral and antero-posterior directions. Placement of the ball is not very accurate because measurement results are not used for calibration, and it is difficult place a ball in an exact position in a space.

Because the ball is very close to the true sphere, it is not necessary to measure whole of the ball for the test. However, it is recommended that at least half of the ball should be measured at each location. Therefore, when the scanning volume is too small for locating the ball at locations specified in Fig. 4, the location was adjusted, and the adjustment was reported.

\subsubsection{Head scanner}

Seven locations as shown in Fig. 4B were selected for measurement. In determining these locations, we have assumed that the scanning volume is ranged from $300 \times 300 \times 300$ [mm] to $400 \times 400 \times 400$ [mm], and tried to cover the volume. The measurer decides the center of the scanning volume. Location \#1 is the center of the scanning volume. Locations \#2 and \#3 are higher or lower than the center by $80 \mathrm{~mm}$, respectively. Locations \#4 and \#5 are anterior or posterior to the center by $80 \mathrm{~mm}$. Locations \#6 and \#7 are 80 $\mathrm{mm}$ to the right or to the left, respectively. Accuracy of the ball placement is within $\pm 10 \mathrm{~mm}$ of the target position for height, lateral and antero-posterior directions.

\subsubsection{Foot scanner}

Four locations as shown in Fig. 4C were selected for measurement. In determining these locations, we assumed that the size of scanning volume is about 350 (antero-posterior direction) x 150 (lateral direction) $\times 200$ (height) $[\mathrm{mm}]$. Accuracy of the ball placement is within $\pm 10 \mathrm{~mm}$ of the target position for height, lateral and antero-posterior directions.

A
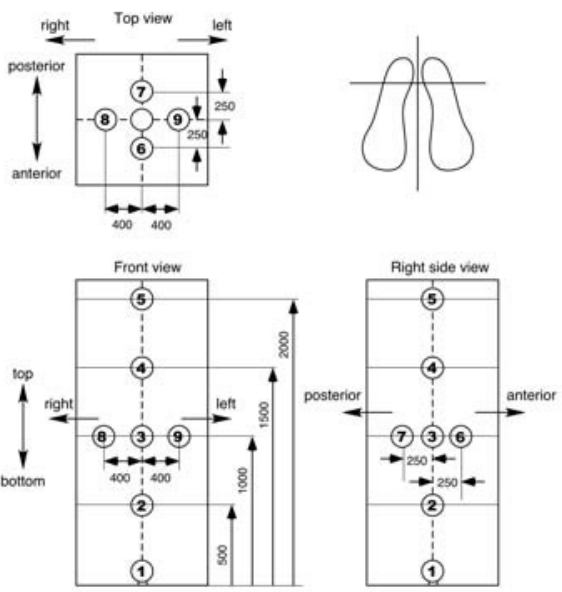

B
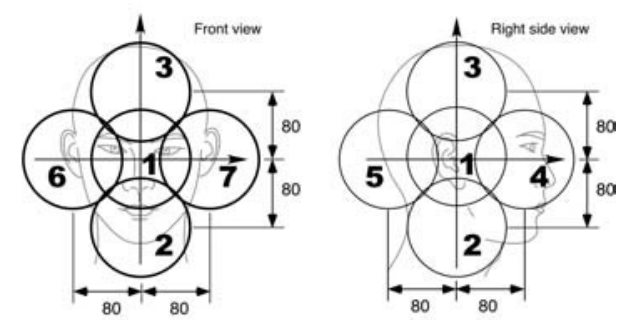

C
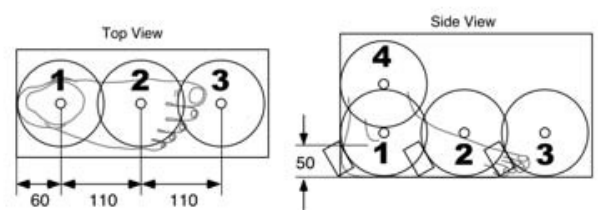

Fig. 4. Locations of measurement of the ball (unit:mm) 


\subsection{Measurement}

The ball was measured at each location specified in Fig. 4. Measurement conditions should be the same with usual human body scanning. The data was exported in tab delimited text format or Wavefront OBJ format.

\section{Measurement of the dummy}

\subsection{Landmarks}

\subsubsection{Whole body scanner}

Forty-seven landmarks listed in Table 1 are used for evaluation. Landmarks \#1-29 are defined in ISO 20685, and shall be used. Landmarks \#30-47 are optional.

\subsubsection{Head scanner}

Fourteen landmarks listed in Table 2 are used for evaluation. Landmarks \#1-9 are defined in ISO 20685, and shall be used. Landmarks \#10-14 are optional.

\subsection{Measurement}

When marker stickers are used to calculate landmark locations, marker stickers are pasted on the dummy before measurement. After each scan, the point cloud data is saved in a tab-delimited text format or Wavefront OBJ format.

\subsubsection{Whole body scanner}

The dummy is placed at a location where a human subject stands. This location is the base location. The dummy is scanned 10 times. After each scan, location of dummy is slightly moved to simulate the variation in the position of human subjects. Variation in location includes antero-posterior and lateral translations and rotational differences. The following locations are used: (1) the base position, (2) $10 \mathrm{~mm}$ anterior to the base location, (3) $10 \mathrm{~mm}$ posterior to the base location, (4) $10 \mathrm{~mm}$ right to the base location, (5) $10 \mathrm{~mm}$ left to the base location, (6) rotate anticlockwise: place only the right heel anterior to the base location by $10 \mathrm{~mm}$, (7) rotate clockwise: place only the left heel anterior to the base location by 10 $\mathrm{mm}$, (8) $10 \mathrm{~mm}$ anterior to the base location, and rotate anticlockwise as the $6^{\text {th }}$ position, (9) $10 \mathrm{~mm}$ posterior to the base location, and rotate clockwise as the $7^{\text {th }}$ position, (10) base location. Point cloud data measured at each location are saved in tab-delimited text format, or Wavefront OBJ format.

\subsubsection{Head scanner}

The dummy is scanned 10 times. The following locations are used: (1) base location (center of the scanning volume of a head scanner), (2) $10 \mathrm{~mm}$ anterior to the base location, (3) $10 \mathrm{~mm}$ posterior to the base location, (4) $10 \mathrm{~mm}$ right to the base location, (5) $10 \mathrm{~mm}$ left to the base location, (6) rotate anticlockwise (procedure of placement is specified in the protocol), (7) rotate clockwise (procedure of placement is specified in the protocol), (8) $10 \mathrm{~mm}$ anterior to the base location, and rotate anticlockwise as the $6^{\text {th }}$ position, (9) $10 \mathrm{~mm}$ posterior to the base location, and rotate clockwise as the $7^{\text {th }}$ position, (10) base location.

Table 1

Evaluated landmarks: whole body scanners

\begin{tabular}{|c|c|c|c|}
\hline 1 & Vertex (top of head) & 25 & Tibiale, L \\
\hline 2 & Tragion, $\mathrm{R}$ & 26 & Lateral malleolus, $\mathrm{R}$ \\
\hline 3 & Tragion, $\mathrm{L}$ & 27 & Lateral malleolus, L \\
\hline 4 & Infraorbitale, $\mathrm{R}$ & 28 & Suprapateralle, R \\
\hline 5 & Infraorbitale, L & 29 & Suprapateralle, L \\
\hline 6 & Glabella & 30 & Side neck point, $\mathrm{R}$ \\
\hline 7 & Sellion & 31 & Side neck point, $\mathrm{L}$ \\
\hline 8 & Menton & 32 & Juglar point \\
\hline 9 & Opisthocranion & 33 & Ant. axilla point, $R$ \\
\hline 10 & Cervicale & 34 & Ant. axilla point, $\mathrm{L}$ \\
\hline 11 & Acromioale, $\mathrm{R}$ & 35 & Post. axilla point, $\mathrm{R}$ \\
\hline 12 & Acromioale, L & 36 & Post. axilla point, $\mathrm{L}$ \\
\hline 13 & Mesosternale & 37 & Omphalion \\
\hline 14 & Thelion, R & 38 & Trochanterion, $\mathrm{R}$ \\
\hline 15 & Thelion, L & 39 & Trochanterion, L \\
\hline 16 & Iliocristale, $\mathrm{R}$ & 40 & Buttock point, $\mathrm{R}$ \\
\hline 17 & Iliocristale, $\mathrm{L}$ & 41 & Buttock point, L \\
\hline 18 & Ant. sup. iliac spine, $\mathrm{R}$ & 42 & Radiale, $\mathrm{R}$ \\
\hline 19 & Ant. sup. iliac spine, L & 43 & Radiale, $\mathrm{L}$ \\
\hline 20 & Stylion, R & 44 & Mid patella, $\mathrm{R}$ \\
\hline 21 & Stylion, L & 45 & Mid patella, L \\
\hline 22 & Ulnar stylion, $\mathrm{R}$ & 46 & Sphyrion, R \\
\hline 23 & Ulnar stylion, L & 47 & Sphyrion, L \\
\hline 24 & Tibiale, $\mathrm{R}$ & & \\
\hline
\end{tabular}

Table 2

Evaluated landmarks: head scanners

\begin{tabular}{|r|l|r|l|r|l|}
\hline 1 & Vertex & 6 & Glabella & 52 & Gonion, L \\
\hline 2 & Tragion, R & 7 & Sellion & 53 & Zygion, R \\
\hline 3 & Tragion, L & 8 & Menton & 54 & Zygion, L \\
\hline 4 & Infraorbitale, R & 9 & Opisthocranion & 55 & Pogonion \\
\hline 5 & Infraorbitale, L & 51 & Gonion, R & \multicolumn{2}{|c}{} \\
\cline { 1 - 3 } & & & \multicolumn{2}{|c}{} \\
\cline { 1 - 3 } & &
\end{tabular}




\section{Quality parameters}

\subsection{The ball}

Procedure for calculating quality parameters is as follows: (1) the measured data was imported to Geomagic studio (Geomagic Inc.), and data points that do not belong to the ball surface were eliminated manually. (2) Coordinates of the center of the best-fit sphere are calculated using the least squares method, by software developed for this purpose. The distance from each data point to the center of the best-fit sphere (radius) is calculated, and the diameter of the best-fit sphere is obtained as the mean of these distances. (3) Calculate the error of diameter measurement (PS) as the diameter of the best-fit sphere minus the true diameter given by CMM. (4) Calculate the following statistics of the distances from data points to the center of the best-fit sphere: $\mathrm{N}$, mean, standard deviation (SD of radius), minimum, maximum, P1, P5, P50, P95, P99 values. (5) Error of spherical form measurement (PFS) is calculated as the maximum distance minus minimum distance.

As quality parameters, the maximum error and median error are calculated:

The maximum error $=\mathrm{PS}_{\mathrm{Max}}+\mathrm{PFS}_{\mathrm{Max}}$,

Average error $=\mathrm{PS}_{\text {Median }}+\mathrm{PFS}_{\text {Median, }}$

where $\mathrm{PS}_{\mathrm{Max}}$ is the maximum PS among the nine/seven/four locations, and $\mathrm{PFS}_{\mathrm{Max}}$ is the maximum value among the nine/seven/four PFS values. Similarly, $\mathrm{PS}_{\text {Median }}$ and $\mathrm{PFS}_{\text {Median }}$ are the median values. The maximum error represents the worst case in the scanning volume.

\subsection{Landmark locations}

It was not possible to give the true values for landmark locations because the shape of the dummy is too complicated for measuring landmark locations using CMM. Therefore, only the repeatability of landmark locations is evaluated.

Only those landmarks that could be measured in all of 10 scans are evaluated. The procedure is as follows: (1) Landmark coordinates obtained from scan \#1 are used as the base data. (2) For each of other scan data, the coordinate system is transformed to minimize the sum of distances between the corresponding landmarks between the base data and the scan. (3) For each of comparison, for each landmark, the error is calculated as the distance between the corresponding landmark locations. (4) Using nine comparison data, calculate mean, standard deviation and median of errors. (5) For each landmark, report mean error, median error, and standard deviation of error.

\section{Results and Discussions}

\subsection{Availability of test objects}

Tables 3 shows 11 whole body scanners, five head scanners, and four foot scanners evaluated in the present study.

All scanner systems could measure the dummy and export the measured data. However, we could not evaluate one whole body scanner using the ball. We could not export ball measurement using this scanner system, which was due to the software specification rather than the limit of the hardware [13].

\subsection{Measurement of the ball}

Figs. 5 and 6 show examples of evaluation results of three whole body scanners (Scanner 1, Scanner 2, and Scanner 3) based on the ball measurements. Fig. 5 shows PS values at nine locations. PS values are not uniform between nine locations in all scanners. Scanner 1 tends to measure the diameter larger than the true value. Scanner 2 has smaller PS values at locations \#8 and \# 9. Scanner 3 has larger PS value at location \#9. If the worst case is used, performance of scanner 2 is not much different from that of Scanner 1 , but Scanner 2 has PS values closer to 0 than Scanner 1 at locations \#1,\#3-\#7.

Fig. 6 shows PFS and SD of radius in the three whole body scanners. In all scanners the precision is not uniform between nine locations. Scanner 1 has larger variation of precision among the nine locations compared to Scanner 2 and Scanner 3. Scanner 1 has worse results than other scanners in some locations, but has as good results as other scanners in other locations. When PFS is large, SD of radius tends to be also large. However, Fig. 6 also shows that in some locations, PFS is considerably larger than expected from general relation between PFS and S.D. This is because minimum and maximum are sensitive to the existence of irregular values.

Fig. 7 shows examples of measurement results of the ball at location \#2 by three whole body scanners. Measurements at location \#2 by all three scanners have small PS $(<1 \mathrm{~mm})$ and small SD or radius $(<1$ $\mathrm{mm})$. However, smoothness of the measured surface appears different between the three scanners. 
Table 3

Availability of test objects

\begin{tabular}{|c|c|c|c|c|c|c|}
\hline \multicolumn{3}{|c|}{ Scanner system } & \multicolumn{2}{|c|}{ Measurement } & \multirow{2}{*}{$\begin{array}{c}\mathrm{N} \text { of LMs (N } \\
\text { of evaluated } \\
\text { LMs) }\end{array}$} & \multirow[b]{2}{*}{ Comment } \\
\hline $\begin{array}{l}\text { Measured } \\
\text { part }\end{array}$ & Maker & type & Ball & Dummy & & \\
\hline Whole body & Hamamatsu & $\begin{array}{l}\text { Bodyline scanner C9036- } \\
02 \mathrm{~S}\end{array}$ & OK & OK & $47(32)$ & Location \#5: $1900 \mathrm{~mm}$ \\
\hline Whole body & Hamamatsu & $\begin{array}{l}\text { Bodyline scanner C9036- } \\
02\end{array}$ & $\mathrm{OK}$ & OK & $47(35)$ & Location \#5: $1900 \mathrm{~mm}$ \\
\hline Whole body & Hamamatsu & $\begin{array}{l}\text { Bodyline scanner C9036- } \\
01\end{array}$ & OK & $\mathrm{OK}$ & Not evaluated & Location \#5: $1750 \mathrm{~mm}$ \\
\hline Whole body & Cyberware, & WB4 & OK & $\mathrm{OK}$ & Not evaluated & \\
\hline Whole body & Cyberware & WBX & $\mathrm{OK}$ & OK & Not evaluated & \\
\hline Whole body & Cyberware & WB4-6004 & OK & OK & $44(44)$ & \\
\hline Whole body & Vitornics & VITUS smart XXL & OK & OK & $17(17)$ & \\
\hline Whole body & TC2 & & n.a. & OK & Not evaluated & Ball data could not be exported \\
\hline Whole body & Space Vision, & Cartecia BS02-N, 3 pole & OK & OK & Not evaluated & $\begin{array}{l}\text { Location \#5: } 1700 \mathrm{~mm} \text {, Locations \#6 \& } \\
\# 7: 250 \mathrm{~mm} \text {, Locations \#8 \& \#9: } 300 \\
\mathrm{~mm}\end{array}$ \\
\hline Whole body & Space Vision & Cartecia BS02, 3 pole & OK & OK & Not evaluated & $\begin{array}{l}\text { Location \#5: } 1700 \mathrm{~mm} \text {, Locations \#6 \& } \\
\# 7: 250 \mathrm{~mm} \text {, Locations \#8 \& \#9: } 300 \\
\mathrm{~mm}\end{array}$ \\
\hline Whole body & Space Vision & Cartecia BS02, 4 pole & OK & OK & Not evaluated & $\begin{array}{l}\text { Location \#5: } 1700 \mathrm{~mm} \text {, Locations \#6 \& } \\
\# 7: 250 \mathrm{~mm} \text {, Locations \#8 \& \#9: } 300 \\
\mathrm{~mm}\end{array}$ \\
\hline Head & $\begin{array}{l}\text { I-Ware } \\
\text { Laboratory, }\end{array}$ & HSU-01-3 & OK & $\begin{array}{c}\text { not } \\
\text { measured }\end{array}$ & Not evaluated & dummy was not measured \\
\hline Head & $\begin{array}{l}\text { I-Ware } \\
\text { Laboratory, }\end{array}$ & Rennacs 1.0 & OK & $\begin{array}{c}\text { not } \\
\text { measured }\end{array}$ & Not evaluated & dummy was not measured \\
\hline Head & Artec & TDSL 1.1 & OK & OK & Not evaluated & $\begin{array}{l}\text { Hand-held scanner. Dummy was } \\
\text { scanned only once }\end{array}$ \\
\hline Head & Cyberware & PX & OK & OK & $14(14)$ & \\
\hline Head & Cyberware & $3030 \mathrm{RGB} / \mathrm{PS}$ & OK & $\mathrm{OK}$ & $12(12)$ & \\
\hline Foot & $\begin{array}{l}\text { I-Ware } \\
\text { Laboratory }\end{array}$ & Infoot compact & OK & $\begin{array}{c}\text { not } \\
\text { measured }\end{array}$ & Not evaluated & Scan data at location \#4 was not good \\
\hline Foot & $\begin{array}{l}\text { I-Ware } \\
\text { Laboratory }\end{array}$ & Infoot USB IFU-S-01 & OK & $\begin{array}{c}\text { not } \\
\text { measured }\end{array}$ & Not evaluated & \\
\hline Foot & $\begin{array}{l}\text { I-Ware } \\
\text { Laboratory }\end{array}$ & Infoot compact & OK & $\begin{array}{c}\text { not } \\
\text { measured }\end{array}$ & Not evaluated & Scan data at location \#4 was not good \\
\hline Foot & DHRC, AIST & 4D measurement system & OK & $\begin{array}{c}\text { not } \\
\text { measured }\end{array}$ & Not evaluated & Ball was measured during rolling \\
\hline
\end{tabular}

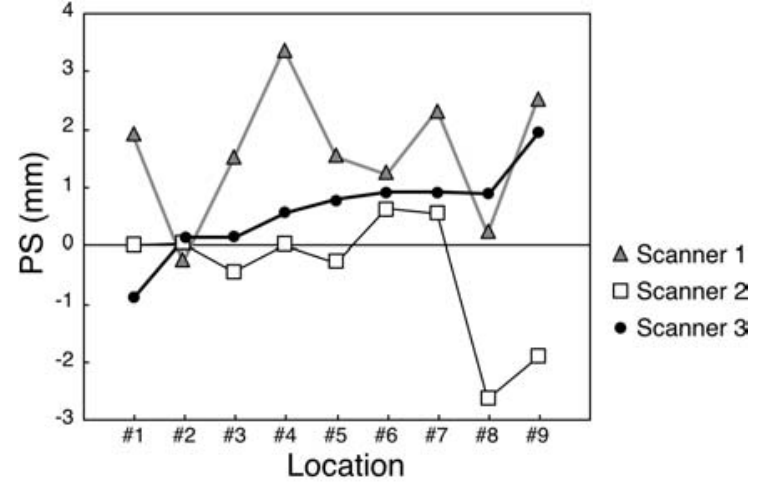

Fig. 5. Examples of evaluation results of whole body scanners based on ball measurement. Error of diameter measurement (PS) at nine locations

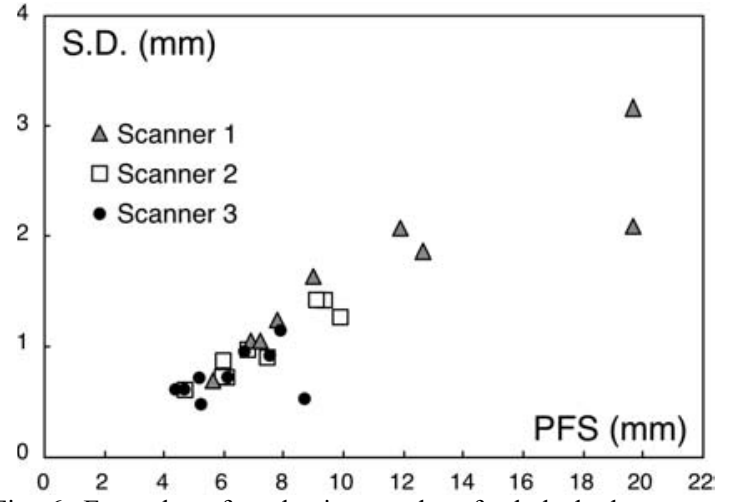

Fig. 6. Examples of evaluation results of whole body scanners based on ball measurement; PFS: error of spherical form mea- 
surement; S.D.: standard deviations of the distance from each data point to the best-fit sphere

Fig. 8 shows ball measurements at locations \#2 and \#7 by Scanner 1. Large PS $(2.29 \mathrm{~mm})$ and large PFS (19.73 mm) values at location \#7 may be due to problems in alignment and/or merging measurements by different cameras.
A

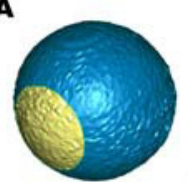

B

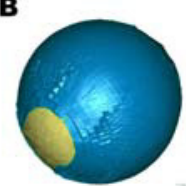

C

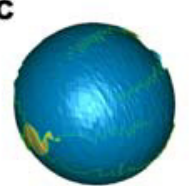

Fig. 7. Ball measurement at location \#2 by three whole body scanners. A: Scanner 1, B: Scanner 2; C: Scanner3.
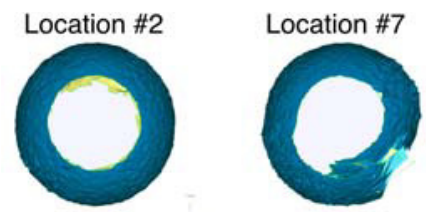

Fig. 8. Ball measurement at locations \#2 and \#7 by whole body scanners, Scanner 1.

\subsection{Measurement of the dummy}

Fig. 9 shows examples of evaluation results of landmark locations for a whole body scanner, Scanner 1. When landmark coordinates obtained from each of scan \#1 to scan \#10 were used as the base data, obtained results can be very different. Therefore, we modified the procedure for calculating quality parameters of landmark errors. Procedure (1) - (3) in section 4.2 were repeated using each of scan \#2 to scan \#10 as the base data, and mean, standard deviation, and median were calculated using $10 \times 9=90$ comparison results. Table 6 shows examples of mean landmark errors for several landmarks by three whole body scanners. There is a strong positive linear relationship between mean error and median error ( $\mathrm{r}>$ 0.9 ). Therefore only mean error or median error may provide enough information.

Scanner 2 and Scanner 3 have several landmarks with large mean errors. Since we used a dummy as the test object, we can ignore factors related to "subject" in Fig. 1. Also, since we evaluate the repeatability using the same marker locations, we can ignore factors related to "measurer skill" in Fig. 1. Therefore the differences between three scanners are due to the tool (hardware accuracy and software performance) and operator skill in using landmarking soft- ware. Locations of marker stickers are automatically detected and labeled by software in Scanner 1, while they are determined and labeled manually by an operator in other two scanners. According to the evaluation results using the ball, performance of Scanner 1 as a tool is not better than other scanners, but repeatability of landmarking is as good as or sometimes better than other two scanners.

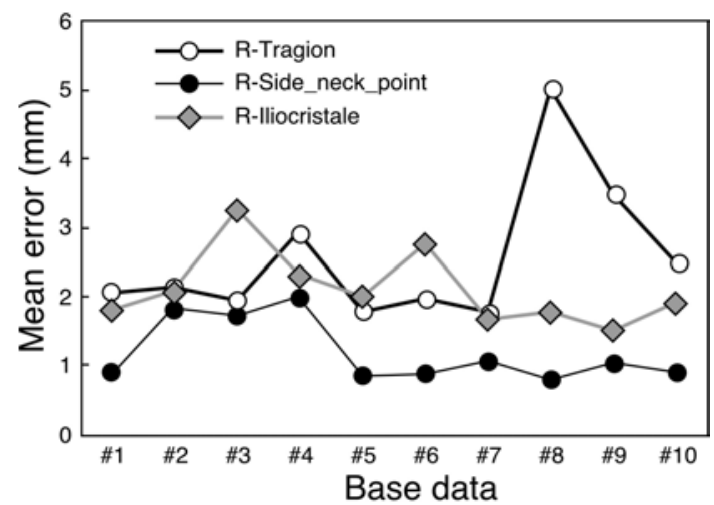

Fig. 9. Example of mean errors in landmarks when different scan is used as the base data. Data obtained by whole body scanner, Scanner 1.

\subsection{Other consideration}

Since body scanners are much more expensive and complex system than traditional tools, they give impression that scan-derived measurements are more accurate than traditional measurements. When the diameter of the ball is measured with a sliding caliper with long jaws, measurements taken by three anthropometrists were all $120.0 \mathrm{~mm}$. This value is closer to the true diameter than any of scanners.

JIS B 7441 and VDI/VDE 2634 series establish an evaluation protocol for the agreement between scanner providers and scanner purchasers. On the other hand, the protocol proposed in this study is intended to establish an agreement between anthropometric database providers and data users. International standards related to the anthropometry established by ISO TC159/WC3/WG1 (ISO 7250-1, ISO 15535, and ISO 20685) are all intended for this purpose. Therefore database providers are expected to validate the accuracy of scanner system they use by conducting the evaluation, and to assure users the accuracy of scan-derived measurements. If the worst-case quality parameter of a scanner is better than the accuracy of traditional tools $(1 \mathrm{~mm})$, it is easy for a database provider to convince data users that the data is accurate. Three of head scanners and all foot scan- 
ners satisfied this condition, but none of whole body scanner used in the present study did. If we use the worst case, the errors would be overestimated in some whole body scanners. Considering the betweenlocation variation of the evaluation results, it may be better to report results from all locations rather than reporting only the worst case. Also it may be better to add quality parameters not sensitive to irregular values.

In the present study, we used all possible pairs of comparisons to calculate repeatability of landmark locations. As another possibility, generalized Procrustes method could be used. This will be considered.

\section{Conclusions}

We proposed a protocol for evaluating the accuracy of $3 \mathrm{D}$ body scanners using two types of test objects. The protocol was examined through a roundrobin test. Proposed test objects were available for all scanners except one, in which measurement of the ball could not be exported. Calibrated ball was useful to evaluate the accuracy of length (accuracy of the diameter of the sphere) and shape (indicator of the smoothness of the measured ball surface). We proposed locations of ball measurement, and considered that providing results from all locations is better than providing only the worst case result. Anthropomorphic dummy of the standard posture with landmarks premarked was useful for evaluating the repeatability of landmark locations.

\section{References}

[1] K.M. Robinette, H. Daanen, E. Paquet, The CAESAR project: a 3-D surface anthropometry survey, Proceedings of Second International Conference on 3-D Digital Imaging and Modeling (1999)

[2] P. Treleaven, Sizing us up, IEEE Spectrum (2004) April: 1719.

[3] RS. Paquette, J.D. Brantley, B.D. Corner, P. Li, and T. Oliver, Automated extraction of anthropometric data from 3D images, Proceedings of the IEA 2000/ HFES 2000 Congress (2000) 6727-730.

[4] J.Y. Wong, A.K. Oh, E. Ohta, A.T. Hunt, G.F. Rogers, J.B. Mulliken, and C.K. Deutsch, Validity and reliability of craniofacial anthropometric measurement of 3D digital photogrammetric images, Cleft Palate Craniofac. J. 45 (2008) 232239.

[5] ISO 20685: 2005 3-D scanning methodologies for internationally compatible anthropometric databases.

[6] M. Mochimaru and M. Kouchi, Statistics for 3D human body forms, SAE Technical Paper (2000) 2000-01-2149

[7] B. Allen, B. Curless, and Z. Popovic, The space of human body shapes: reconstruction and parameterization from range scans. ACM Transactions on Graphics (ACM SIGGRAPH 2003) 22 (2003) 587-594.

[8] JIS B 7441: 2009 Acceptance and reverification tests for coordinate measuring machines $(\mathrm{CMM})$ with non-contacting probing systems.

[9] VDI/VDE 2634 Part 1: 2002 Optical 3-D measuring systems Imaging systems with point-by-point probing.

[10]VDI/VDE 2634 Part 2: 2002 Optical 3-D measuring systems Optical systems based on area scanning.

[11]VDI/VDE 2634 part 3: 2008 Optical 3D-measuring systems Multiple view systems based on area scanning.

[12]S. Sato, S. Osawa, T. Takatsuji, M. Murakami and R. Harada, Test artifacts for the verification of optical digitizers, Key Engineering Materials, 381-382 (2008) 553-556.

[13]Personal communication with the scanner maker

Table 4

Examples of landmarking errors for some landmarks by three whole body scanners (unit: mm)

\begin{tabular}{|c|c|c|c|c|c|c|c|c|c|}
\hline & \multicolumn{3}{|c|}{ Scanner 1} & \multicolumn{3}{|c|}{ Scanner 2} & \multicolumn{3}{|c|}{ Scanner 3} \\
\hline Landmark & Mean & S.D. & Median & Mean & S.D. & Median & Mean & S.D. & Median \\
\hline Tragion, $\mathrm{R}$ & 2.0 & 1.9 & 1.4 & 2.9 & 1.4 & 2.8 & 3.0 & 1.5 & 3.4 \\
\hline Infraorbitale, $\mathrm{R}$ & 1.3 & 1.0 & 1.1 & 2.3 & 1.2 & 1.7 & 2.1 & 1.2 & 2.0 \\
\hline Cervicale & 1.7 & 1.1 & 1.5 & 1.2 & 0.5 & 1.1 & 4.9 & 1.1 & 4.8 \\
\hline Mesosternale & 2.7 & 1.6 & 2.9 & 1.9 & 0.9 & 2.1 & 2.8 & 1.1 & 2.9 \\
\hline Thelion, R & 1.3 & 1.2 & 1.1 & 4.7 & 2.5 & 4 & 1.5 & 0.7 & 1.3 \\
\hline Iliocristale, $\mathrm{R}$ & 1.8 & 1.3 & 1.4 & 1.0 & 0.5 & 1.1 & 2.2 & 0.9 & 2.4 \\
\hline Iliospinale ant., $\mathrm{R}$ & 1.5 & 1.0 & 1.3 & 1.2 & 0.6 & 1.5 & 1.3 & 0.6 & 1.5 \\
\hline Stylion, $\mathrm{R}$ & 2.4 & 0.8 & 2.2 & 1.5 & 1.1 & 1.2 & 2.3 & 1.0 & 2.5 \\
\hline Ulnar stylion, $\mathrm{R}$ & 2.4 & 1.1 & 2.3 & 1.6 & 0.7 & 1.6 & 5.3 & 1.6 & 4.5 \\
\hline Tibiale, $\mathrm{R}$ & 2.8 & 1.4 & 2.4 & 1.3 & 0.7 & 1.3 & 2.6 & 0.6 & 2.5 \\
\hline Side neck point, $\mathrm{R}$ & 0.8 & 0.5 & 0.6 & 5.1 & 4.2 & 5.3 & 2.9 & 1.4 & 2.7 \\
\hline Ant. axilla point, $\mathrm{R}$ & 1.9 & 1.0 & 1.7 & 5.3 & 3.1 & 5.1 & 1.0 & 0.4 & 1.0 \\
\hline Post. axilla point, $\mathrm{R}$ & 2.6 & 1.0 & 2.2 & 2.9 & 1.6 & 2.9 & 1.9 & 1.7 & 1.8 \\
\hline
\end{tabular}

